\title{
Motivações para práticas coletivas na Atenção Básica: percepção de usuários e profissionais
}

\author{
Thaís Lopes Friedrich(a) \\ Xavéle Braatz Petermann ${ }^{(b)}$ \\ Silvana Basso Miolo(c) \\ Hedioneia Maria Foletto Pivetta(d)
}

Friedrich TL, Petermann XB, Miolo SB, Pivetta JMF. Motivations for collective practices in Primary Care: perception of users and professionals. Interface (Botucatu). 2018; 22(65):373-85.

This study aimed to understand the motivations for the development and ongoing group activities in Primary Care in the perception of users and professionals. It is a descriptive research with qualitative methodology, developed in a Health Unit with a Community Health Workers Strategy in a city in southern Brazil. Fifteen patients and five professionals were included in this study, using an intentional sample plus convenience and saturation. The data were collected through individual semi-structured narrative interviews, analyzed using content analysis. The categories found that the subjects motivations comprise interpersonal relations, knowledge construction and comprehensive care. The results reveal the importance of teamwork in Primary Care to health promotion, once it has a positive impact on the clinical, social and emotional conditions of the subjects.

Keywords: Health primary care. Group processes. Motivation.
Este estudo teve por objetivo compreender as motivações para o desenvolvimento e manutenção de ações grupais na Atenção Básica na percepção de usuários e profissionais. Trata-se de pesquisa descritiva com metodologia qualitativa desenvolvida em uma Unidade de Saúde com Estratégia de Agentes Comunitários de Saúde de um município do Sul do Brasil. Participaram 15 usuários e cinco profissionais, sendo a amostra intencional, por conveniência e saturação. Os dados foram coletados mediante entrevista narrativa semiestruturada individual e analisados por meio da análise de conteúdo. As categorias que denotaram as motivações dos sujeitos compreenderam as relações interpessoais, a construção de saberes e o cuidado integral. Os resultados encontrados retrataram a importância do trabalho grupal na Atenção Básica para a promoção da saúde, uma vez que apresentaram impacto positivo sobre as condições clínicas, sociais e afetivas dos sujeitos.

Palavras-chave: Atenção primária à saúde. Processos grupais. Motivação.

\footnotetext{
(a) Universidade Federal de Santa Maria. Av. Roraima $n^{\circ} 1000$, bairro Camobi. Santa Maria, RS, Brasil. 97105-900. thais.friedrich@ yahoo.com.br

(b) Universidade Federal de Santa Maria. Santa Maria, RS, Brasil. xavelepetermann@ gmail.com

(c) Prefeitura Municipal de Santa Maria. Santa Maria, RS, Brasil. sbmiolo@hotmail.com

(d) Departamento de Fisioterapia e Reabilitação, Universidade Federal de Santa Maria. Santa Maria, RS, Brasil. hedioneia@gmail.com
} 


\section{Introdução}

A Atenção Básica $(A B)$ constitui a principal porta de entrada e centro de comunicação da Rede de Atenção à Saúde (RAS) e orienta-se pelos princípios do Sistema Único de Saúde (SUS) - universalidade, integralidade e equidade ${ }^{1}$. Caracteriza-se por um conjunto de ações de promoção da saúde, prevenção de agravos, diagnóstico, tratamento e reabilitação, dirigidas a populações de territórios adstritos ${ }^{1}$.

As Unidades Básicas de Saúde (UBS) localizadas geograficamente próximas aos usuários permitem aos profissionais de saúde a melhor compreensão sobre o contexto cultural, social e econômico em relação aos usuários adscritos a essas Unidades. Assim sendo, os profissionais vinculados a essas UBS assumem papel fundamental para a melhoria das condições de saúde da população de sua abrangência ${ }^{1}$. Nas UBS é prevista a implantação da Estratégia de Agentes Comunitários de Saúde (EACS), como um meio para a reorganização inicial da $A B^{1}$. As UBS com Estratégia de Agentes Comunitários de Saúde, por sua vez, consistem em UBS tradicionais que contam com as ações dos Agentes Comunitários de Saúde coordenados por um enfermeiro1.

Alguns profissionais, como o fisioterapeuta, não compõem a equipe mínima na $A B$, entretanto, $a$ proposta atual da Política Nacional de Atenção Básica (PNAB) é que a inserção deste profissional nesse nível de atenção ocorra por meio dos Núcleos de Apoio à Saúde da Família (NASF) ${ }^{1}$.

Os estudos apontaram que a inserção de outros profissionais, além daqueles previstos na equipe mínima, como fisioterapeuta, fonoaudiólogo e terapeuta ocupacional na AB tem como objetivo o fortalecimento desse nível de atenção, uma vez que aumenta a resolutividade do sistema e contribui para a integralidade no cuidado ${ }^{2-7}$. Uma das modalidades de atuação dos profissionais de saúde no nível primário é por meio do trabalho grupal, com ações de educação em saúde e atividade física ${ }^{8}$.

Os grupos de promoção da saúde envolvem conhecimentos, habilidades e atitudes, que compreendem aspectos emocionais, sociais e biológicos, não se configurando apenas como um somatório de pessoas, mas como uma nova entidade com objetivos compartilhados ${ }^{9}$, consistindo em instrumentos de intervenção coletiva e interdisciplinar, com a finalidade de construir relações socias cooperativas para o desenvolvimento contínuo da autonomia dos seus integrantes ${ }^{10}$. Compreendem, também, um espaço privilegiado para a construção da rede de atenção, efetivando a participação popular e promovendo a educação em saúde ${ }^{11}$.

A partir do exposto, pretendeu-se identificar os motivos pelos quais os usuários e profissionais do sistema de saúde aderiram às ações grupais e os motivos que os fizeram permanecer em tais atividades na $A B$. Entende-se, assim, a relevância de se compreender a constituição destes grupos, para o planejamento dessas ações, no sentido de torná-las mais efetivas e resolutivas ${ }^{12}$.

Justifica-se o desenvolvimento da pesquisa na referida UBS pelas vivências práticas da Fisioterapia, por intermédio do Programa de Reorientação da Formação do Profissional da Saúde e do Programa de Educação pelo Trabalho para a Saúde junto à equipe de saúde desta UBS.

Entretanto, mesmo que haja profissionais que, na legislação atual, não integrem a equipe mínima, como o fisioterapeuta, o fonoaudiólogo e o terapeuta ocupacional, existem algumas iniciativas de trabalho em grupo que envolvem a educação em saúde e o movimento humano, de forma terapêutica, coordenadas por estes profissionais na AB. Com essa característica de prática grupal, este estudo teve como objetivo compreender as motivações para o desenvolvimento e a manutenção de atividades em grupo na $A B$ na percepção de usuários e profissionais.

\section{Metodologia}

A pesquisa utilizada foi a do tipo descritiva, transversal de cunho qualitativo, que buscou compreender as motivações para o desenvolvimento e a manutenção de atividades em grupo na $A B$ na percepção de usuários e profissionais. De acordo com Minayo ${ }^{13}$, a pesquisa qualitativa caracteriza-se pela preocupação com a profundidade dos significados dos dados obtidos, e não com a generalização dos mesmos.

Assim sendo, os dados foram coletados em um grupo de promoção à saúde de uma UBS com EACS da rede de atenção à saúde de um município do Sul do Brasil, no período de março a julho de 2015. 
A amostra foi intencional, por conveniência e saturação, sendo composta por 15 usuários e cinco profissionais os quais aceitaram participar da pesquisa. A amostragem por saturação é utilizada para estabelecer o tamanho final da amostra de uma pesquisa, cessando a captação de novos dados ${ }^{14}$. As narrativas dos novos sujeitos da pesquisa pouco acrescentariam ao material já obtido, não contribuindo de forma significativa para aprimorar a reflexão fundamentada nos dados até o momento coletados $^{14}$.

Para participar do estudo os sujeitos poderiam ser de ambos os sexos, de todas as idades e que tivessem participando das atividades em grupo promovidas pelos trabalhadores da UBS, em parceria com o PET-Saúde, por, no mínimo, três meses, tempo considerado suficiente, segundo Augusto et al. ${ }^{15}$, para que os sujeitos possam narrar sua percepção a respeito das ações grupais. Foi excluído um sujeito por apresentar dificuldade de compreensão e expressão dos questionamentos, no momento da realização da entrevista, e que não permitiria o levantamento de informações pertinentes à pesquisa.

As ações em grupo tiveram início em 2007, ocorrendo em duas vezes na semana. Cada encontro tinha a duração de uma hora, com a participação de vinte a cinquenta usuários. As ações compreenderam o diálogo, o exercício físico, as atividades lúdicas e o relaxamento. O grupo era coordenado pelo fisioterapeuta, em parceria com o Técnico de Enfermagem e os Agentes Comunitários de Saúde.

A partir do Quadro 1 pôde-se perceber que o tempo de participação dos usuários no grupo era de três a 96 meses, sendo, em sua maioria, do sexo feminino, idosos e aposentados. Os profissionais que participaram das atividades grupais na UBS foram o Fisioterapeuta, o Técnico de Enfermagem e os três Agentes Comunitários de Saúde (ACS), com tempo de participação em tais atividades de 24 a 66 meses.

Para preservar a identidade dos sujeitos envolvidos na pesquisa, optou-se pelo uso de pseudônimos. Preferiu-se não utilizar nomes abstratos, mas sim que tivessem algum significado para evidenciar os participantes envolvidos nas ações em grupo. Para isso, foram adotados nomes de pedras preciosas para os usuários e de estrelas, para os profissionais. Justifica-se o uso desses pseudônimos, porque representavam o brilho de cada sujeito para o grupo, sendo que estes pseudônimos foram escolhidos por meio de um sorteio.

Quadro 1. Caracterização dos sujeitos

\begin{tabular}{|l|c|l|l|c|}
\hline \multicolumn{1}{|c|}{ Nome } & Idade (anos) & \multicolumn{1}{c|}{ Sexo } & \multicolumn{1}{c|}{ Profissão } & Tempo de participação (meses) \\
\hline Ágata & 64 & Feminino & Aposentada & Sete \\
\hline Jaspe & 66 & Feminino & Aposentada & 24 \\
\hline Safira & 69 & Feminino & Aposentada & 96 \\
\hline Jade & 61 & Feminino & Aposentada & Quatro \\
\hline Apatita & 51 & Feminino & Comerciante & 12 \\
\hline Cristal & 75 & Feminino & Aposentada & 12 \\
\hline Âmbar & 69 & Feminino & Aposentada & 36 \\
\hline Pérola & 57 & Feminino & Cuidadora & Três \\
\hline Malaquita & 73 & Feminino & Aposentada & 36 \\
\hline Rubi & 54 & Feminino & Doméstica & 48 \\
\hline Ônix & 55 & Feminino & Doméstica & 12 \\
\hline Diamante & 56 & Feminino & Aposentada & 24 \\
\hline Brilhante & 67 & Feminino & Aposentada & 36 \\
\hline Quartzo & 66 & Masculino & Aposentado & 24 \\
\hline Citrino & 74 & Masculino & Aposentado & 96 \\
\hline Luna & 42 & Feminino & Fisioterapeuta & 24 \\
\hline Sirius & 56 & Masculino & Técnico de enfermagem & 54 \\
\hline Meissa & 36 & Feminino & Agente comunitária de saúde & 60 \\
\hline Rana & 55 & Feminino & Agente comunitária de saúde & 36 \\
\hline Mira & 45 & Feminino & Agente comunitária de saúde & \\
\hline
\end{tabular}


O instrumento de coleta de dados utilizado foi a entrevista narrativa semiestruturada individual elaborada pelos autores e validada com população semelhante que não fez parte da pesquisa. $O$ roteiro da entrevista é um instrumento usado para orientar uma conversa com finalidade, que é a entrevista ${ }^{13}$. A entrevista foi realizada individualmente, conforme disponibilidade de horário dos participantes, e gravada em equipamento de áudio, na UBS, no intuito de absorver, ao máximo, as narrativas dos participantes, sem tempo pré-definido, para que os entrevistados tivessem liberdade de pronunciamento, conforme desejado.

A entrevista realizada com os usuários foi constituída de eixos temáticos, que contemplaram dados sociodemográficos para a caracterização dos participantes do grupo, tais como, sexo, idade, profissão, assim como dados sobre a inserção e a percepção do grupo, tempo de participação no grupo, maneira como ficou sabendo do grupo, dinâmica do grupo, escolha e avaliação das atividades, motivos de adesão e permanência nas ações em grupo, implicações na saúde, dificuldades para participação no grupo e atuação dos profissionais no grupo. Além das temáticas descritas, a entrevista dos profissionais de saúde contemplou os motivos para desenvolver e permanecer nas ações em grupo, caracterização do trabalho, tempo de participação e dinâmica do grupo, planejamento e avaliação das ações, dificuldades na realização do grupo, percepção sobre as implicações na saúde dos usuários e atuação dos profissionais.

A análise dos dados foi realizada por meio da análise de conteúdo das entrevistas narrativas dos usuários e profissionais. Existem várias modalidades de análise de conteúdo, sendo uma delas a análise temática, utilizada neste estudo, pois é considerada a mais apropriada para pesquisas qualitativas em saúde ${ }^{13}$.

A análise temática busca descobrir os núcleos de sentido que compõem uma comunicação, cuja presença ou frequência adquirem algum significado para o objeto estudado ${ }^{13}$. Ela compreende três etapas $^{13}$ : pré-análise (compreende a escolha dos documentos a serem analisados e a retomada das hipóteses dos objetivos iniciais da pesquisa); exploração do material (consiste na categorização, sendo este um processo de redução do texto às palavras ou expressões mais significativas); tratamento dos resultados obtidos e interpretação (são propostas inferências e realizadas interpretações, interrelacionando-as com o quadro teórico inicialmente desenhado $)^{13}$. Nesse sentido, as narrativas dos usuários e profissionais foram analisadas em conjunto, na tentativa de permitir uma análise uníssona das ações grupais.

O estudo foi aprovado pelo Comitê de Ética da Instituição de Ensino Superior à qual os autores estão vinculados e está de acordo com a Resolução n 466, de 12 de dezembro de 2012, do Conselho Nacional de Saúde.

\section{Resultados e discussão}

Para melhor organização e clareza dos resultados, apresenta-se a categorização que emergiu da análise de conteúdo e que denotou as motivações para o desenvolvimento e a manutenção de ações em grupo na $A B$, na percepção dos usuários e profissionais.

\section{Motivações para a prática de atividades em grupo na percepção dos usuários e profissionais}

As análises das narrativas revelaram três categorias. A primeira foi denominada de: "o grupo como meio para a construção de relações interpessoais"; a segunda: "o grupo como elemento para a construção de saberes", e a terceira: "o grupo como espaço para o cuidado integral". Estas narrativas trouxeram os motivos para a adesão e a permanência, na perspectiva uníssona dos usuários e dos profissionais das ações grupais.

As categorias, bem como os elementos que as elegeram, apresentaram-se inter-relacionadas no contexto grupal. Neste contexto, a Figura 1 ilustrou as motivações para o desenvolvimento das ações grupais. 


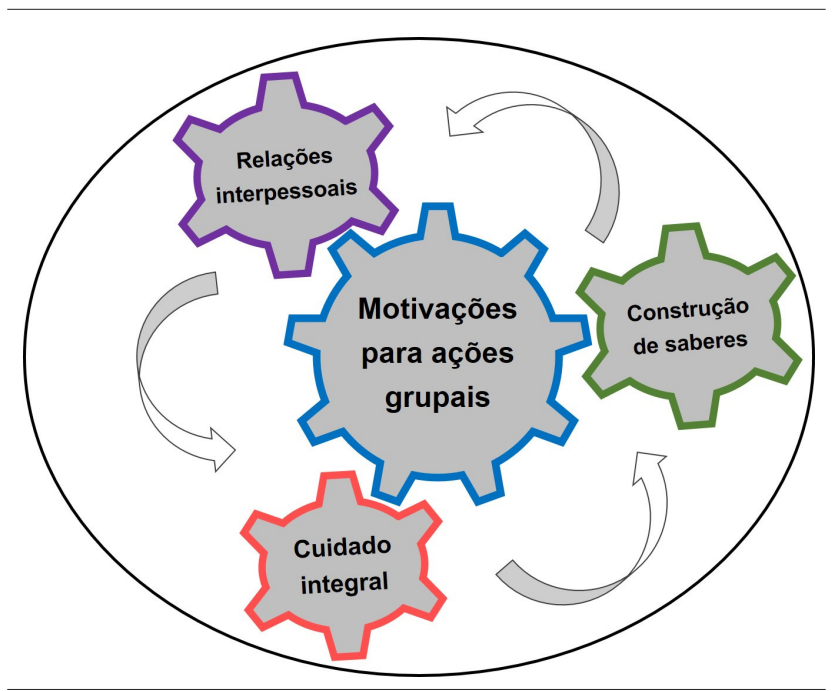

Figura 1. Motivações para o desenvolvimento de ações grupais.

\section{O grupo como meio para a construção de relações interpessoais}

A partir da convivência entre os sujeitos no grupo, houve a criação de laços de afetividade e a construção do vínculo, os quais tornaram-se facilitadores para o processo de troca de experiências e aprendizado. Assim, os elementos que elegeram essa categoria foram, fundamentalmente, os 'laços afetivos' e o 'vínculo' que emergiram das relações oriundas do espaço grupal.

Os 'laços afetivos' permeiam as relações entre os sujeitos, por meio de sentimentos e emoções, esses laços podem ser compreendidos como a manifestação de sentimentos de estima a alguém, de simpatia, de amizade que faz uma relação ter cumplicidade ${ }^{16}$.

A partir dos relatos, notou-se que os 'laços afetivos' entre os sujeitos do grupo constituíram um importante fator de motivação em tais atividades:

Têm conversas, no início do grupo, a gente pede como está a pessoa, que seja bem bem-vinda, às vezes, um carinho, um abraço, a pessoa melhora. (Apatita)

[...] encontrar colegas, trocar ideia, se dar um abraço, essa troca, além do físico [...]. (Âmbar)

A relação entre nós é de respeito, de amizade, de companheirismo, e entra também o pessoal, que também é a mesma coisa, e eu acho que se passa muita motivação [...]. (Mira)

Os 'laços afetivos' descritos pelos usuários e profissionais envolveram o carinho, o respeito, a amizade e o companheirismo, os quais retrataram a importância dos aspectos afetivos na adesão e permanência dos usuários e profissionais nas ações grupais na $A B$. Em pesquisa realizada por Bittar et al. ${ }^{17}$, o grupo foi considerado pelos usuários e profissionais como lugar para o afeiçoamento entre as pessoas. Outro estudo também encontrou resultados semelhantes, em que os grupos se apresentaram como lugares de informação, mas, ao mesmo tempo intervenções cheias de afeto ${ }^{18}$.

Desse modo, percebeu-se que na $A B$, as ações em saúde, principalmente de promoção da saúde, requereram tecnologias de baixa densidade. Apesar do conhecimento profissional e de todo $o$ repertório para o desenvolvimento de tais atividades, os aspectos de cunho emotivo prevaleceram.

Mediante o exposto, o "vínculo", outro dispositivo necessário às ações em saúde, é construído em um processo relacional, que transcorre ao longo do tempo de convivência no grupo, e compreende 
a ligação de confiança entre os sujeitos, garantindo a continuidade do cuidado. Segundo a Política Nacional de Atenção Básica (PNAB) ${ }^{1}$, o vínculo consiste na construção de relações de afeto e confiança entre os sujeitos, construído ao longo do tempo, permitindo aprofundar o processo de corresponsabilização pela saúde". Neste estudo, foi possível perceber que o "vínculo" pode ter sido construído nas relações profissional-usuário, usuário-usuário e profissional-profissional, constituindose, essencialmente, um vínculo social.

O "vínculo" entre profissional-usuário compreendeu uma parceria instituída, em prol do fortalecimento do cuidado, como pôde ser observado nas falas:

[...] ela é tipo aquela mãe amorosa [...] tem uma palavra amiga para tudo [...]. Durante os exercícios é muito bom, sempre alegre, divertida, ela é companheira mesmo, nem parece a professora, parece uma de nós. (Pérola)

[...] eles sentem a energia do profissional que chega e chega para fazer a diferença, é isso que faz eles voltarem, eles sentem o prazer do profissional que está ali com eles [...], é uma relação muito horizontal, [...] e isso fortalece o vínculo, e não é aquela coisa do profissional no andar de cima e o usuário no andar de baixo. (Luna)

Os pronomes "ela" e "eles", que emanaram das vozes dos usuários e profissionais, referiram-se ao profissional da equipe e aos próprios participantes do grupo. Conforme descrito, o 'vínculo' baseado em uma relação horizontal entre tais sujeitos é um importante motivo de adesão e permanência destes no grupo. A valorização do outro como sujeito ativo é fundamental para a efetivação dos objetivos das ações desenvolvidas em âmbito coletivo. Um estudo relatou que, quando o profissional senta-se ao lado, ouve as dificuldades dos usuários e constrói, em conjunto, as soluções para os seus problemas, faz com que a equipe de saúde seja vista como parceira e este fator fortalece o cuidado ${ }^{19}$. A partir do exposto, notou-se que as relações de 'vínculo' entre tais sujeitos interferiram de maneira significativa nas ações desenvolvidas e foram valorizadas no contexto do grupo.

O vínculo é considerado uma conquista, não é um acontecimento imediato e quanto mais forte o vínculo, maior é a troca de saberes entre tais sujeitos, gerando ações de saúde mais resolutivas ${ }^{20}$. Uma pesquisa realizada com um grupo de atividade física com idosos identificou que ter bons profissionais foi um fator que motivou tais sujeitos ${ }^{12}$. Outro estudo sobre barreiras e facilitadores para a prática de atividade física em grupo de idosas identificou como facilitador as atitudes profissionais ${ }^{19}$. Dessa forma, conforme as narrativas e os estudos citados ${ }^{12,10}$, a relação de 'vínculo' entre usuários e profissionais apresentou-se como um importante aspecto na motivação em ações grupais.

Ainda, na perspectiva do 'vínculo', este dispositivo estabeleceu-se, densamente, entre os usuários na convivência no grupo, e foi fortalecido e ampliado no decorrer do tempo. As narrativas, que seguem, demonstraram esse achado:

[...] uma conversa com a outra, uma dá ânimo para a outra, incentivando a ficar no grupo, incentivando a vim para o grupo, [...], a gente tem outra qualidade de vida. (Ágata)

[...] uma ajuda a outra e fica aquela união, [...] vai conversando, pela amizade também [...]. (Brilhante)

[...] pela convivência e que é um grupo bom, é um grupo assim que as pessoas estão bem, estão alegres, conversam, se comunicam, hoje em dia os grupos estão muito fechados, as pessoas ficam muito na sua e aqui a gente vê, sempre conversam, dizem alguma coisa, se expressam [...]. (Rubi)

Como foi possível perceber, o "vínculo" constituiu a maneira como os usuários relacionaram-se no contexto do grupo, por meio da conversa, do incentivo e da convivência, descrito nos relatos, e em razão disso, definiu-se como motivo de adesão e permanência dos colaboradores no grupo. 
Um dos efeitos das práticas grupais, de acordo com a pesquisa de Ferreira Neto et al. ${ }^{18}$, é exatamente o de criação de espaços de sociabilidade, já que os usuários, com vínculos sociais e familiares estreitos, encontram formas de estabelecer laços de amizade e solidariedade ${ }^{18}$. Na presente pesquisa, também, notou-se a existência do vínculo social entre os usuários, confirmado pelas narrativas que denotaram sentimentos de afeto e de confiança entre eles. Tal relação estabelecida entre os participantes do grupo, sem dúvida, tiveram o efeito de fortalecer as ações, proporcionando o acolhimento no ambiente grupal.

O "vínculo" entre os profissionais, também, foi identificado nas narrativas dos participantes deste estudo, como importante para o trabalho em conjunto no grupo e para o funcionamento deste, pois ampliou o entendimento sobre a atuação no grupo. Os relatos demonstraram essa relação:

[...] todos os profissionais trabalham juntos com a mesma função para um resultado só [...]. (Meissa)

Cada profissional tem aquela partezinha, tem aquele apoio, dá um pouquinho de si para formar uma grande equipe. (Rana)

[...] o entrosamento com o grupo, a gente conversa, a gente brinca, a gente ri, mexe o corpo, mexe a mente, é um todo, é muito importante [...]. (Âmbar)

As narrativas denotaram que, quando os profissionais trabalhavam em parceria e harmonia, tornavam o grupo mais unido e os usuários percebiam o vínculo e o comprometimento, favorecendo a adesão e a permanência dos usuários em tais ações.

Um estudo de Viegas et al. ${ }^{21}$, com o objetivo de compreender a construção das práticas de integralidade em saúde no trabalho das equipes de Saúde da Família e de gestores de três municípios de Minas Gerais, referiu a necessidade de integração entre os diferentes profissionais para a atenção integral dos indivíduos. Apesar de o estudo citado ${ }^{21}$ ter sido realizado em outra realidade e no contexto da Saúde da Família, os resultados também demonstraram a importância do vínculo entre os profissionais para promoverem uma atenção integral.

\section{O grupo como elemento para a construção de saberes}

No espaço grupal, a partir das relações entre os sujeitos, ocorreram troca de experiências e aprendizagem, oportunizando o empoderamento dos usuários sobre o cuidado com a sua saúde. Os elementos que elegeram essa categoria puderam compreender a "troca de experiência", o "aprendizado" e o "empoderamento do usuário" quanto ao cuidado com sua saúde.

A "troca de experiência" entre os sujeitos no grupo envolveu o intercâmbio de saberes sobre os aspectos relacionados ao cotidiano, com enfoque na saúde, como demonstrado pelos depoimentos:

[...] tem um dia da semana que a gente conversa, faz aquela integração, cada um conta a sua experiência, passa uma mensagem boa. (Rubi)

[...] são trabalhados alguns temas que servem para eles refletirem sobre o dia-a-dia. [...] o ACS [...], além de ser uma capacitação para ele, vai ter a troca de experiência com os usuários [...]. (Luna)

A partir das narrativas percebeu-se que, nas ações de educação em saúde desenvolvidas no grupo, os sujeitos tiveram a oportunidade de trocar experiências, e com isso, ampliar seu entendimento sobre determinados assuntos ligados à saúde. Um estudo realizado sobre atividades em grupo, como estratégia de promoção da saúde na senescência destacou que tais atividades permitiram a troca de experiências entre os participantes, o que favoreceu o aprendizado ${ }^{17}$. Ferreira Neto et al. ${ }^{18}$ relataram que um dos efeitos das práticas grupais é a troca de conhecimentos entre os sujeitos envolvidos 
no grupo, possibilitando a construção coletiva de saberes. Percebeu-se a importância da "troca de experiências" entre os indivíduos no grupo, o que permitiu a construção conjunta de saberes.

O 'aprendizado', como consequência, caracterizou-se pela apropriação de conhecimentos significativos para os sujeitos, possibilitando um impacto ou uma mudança na vida dos usuários ou na prática dos profissionais de saúde, conforme as falas:

[...] eu não sabia o que tinha que comer, o certo e o errado, para controlar a pressão [...] para o diabetes. [...] vi resultados bons, eu estou aprendendo muita coisa sobre a saúde [...]. Os remédios, que, às vezes, a gente não tomava no horário, agora a gente toma certo. (Ônix)

[...] ter um maior aprendizado, porque eu já trabalho com a comunidade, ter um algo a mais para acrescentar, para poder orientar nas visitas, alguns exercícios que a gente aprendeu no grupo. (Meissa)

Notou-se que a aprendizagem se deu com assuntos relacionados à alimentação, ao controle de doenças crônicas, ao uso correto de medicamentos e à prática de exercício físico. O "aprendizado", neste contexto, também foi significativo para o ACS, pois ele foi multiplicador de conhecimento nas suas ações na comunidade, visto que trocou e compartilhou experiências no convívio com outros profissionais, o que configurou ações importantes na educação permanente em saúde.

O processo de aprendizado ocorrido como resultado do trabalho grupal envolve componentes que facilitam a modificação de comportamentos direcionados à promoção da saúde ${ }^{22}$. Um estudo realizado por Almeida et al. ${ }^{23}$ buscou investigar o processo de aprendizado em um grupo de diabéticos desenvolvido em uma UBS de Belo Horizonte, e mostrou que a maioria dos entrevistados referiu-se ao grupo como espaço de aprendizagem do cuidado com o diabetes, sendo que este aprendizado aconteceu a partir da comunicação e da interação entre os participantes do grupo, sendo valorizados o conhecimento e a experiência de cada um. Os autores ${ }^{23}$, também, constataram que a aprendizagem em grupo se deu por meio de orientações, informações, esclarecimento de dúvidas e compartilhamento de experiências, o que convergiu com os achados desta pesquisa, pois os entrevistados, também, se remeteram ao grupo como um espaço potencial para a construção do aprendizado sobre saúde.

Embora o estudo ${ }^{23}$, em questão, tratasse de um grupo em torno de uma condição específica, o diabetes, os resultados estavam de acordo com os encontrados na presente pesquisa, uma vez que os entrevistados, também, se remeteram ao grupo como um espaço potencial para a construção do aprendizado sobre a saúde.

Nesse sentido, pôde-se caracterizar como "empoderamento do usuário" o processo de aquisição de conhecimento capaz de gerar autonomia perante o seu cuidado, o que foi descrito pelas narrativas:

[...] o papel do fisioterapeuta, também, é esse, empoderar as pessoas para que elas sejam responsáveis pelo seu cuidado [...]. (Luna)

[..] o nosso grupo, assim, é muito divertido, [...]. Nosso grupo desempenha um papel excelente. [...] quando a fisioterapeuta não está, a gente, também, faz o grupo sozinho, tem uma residente ajudando a gente [...]. (Jade)

As falas demonstraram que um dos papéis dos profissionais de saúde, dentre eles, o fisioterapeuta, no grupo, é de empoderar os usuários pelo cuidado com a sua saúde. Neste contexto, o empoderamento é o processo no qual as pessoas adquirem o domínio sobre as suas vidas, apreendendo conhecimentos, para serem capazes de tomar decisões acerca de sua saúde ${ }^{24}$. Em um estudo, Santos et al. ${ }^{22}$ destacaram que os grupos de promoção à saúde têm como papel a potencialização das capacidades dos sujeitos, isto é, torna-os capazes de realizarem mudanças de comportamento e de atitude direcionados ao desenvolvimento da autonomia e o enfrentamento das condições geradoras de sofrimentos evitáveis/desnecessários, o que pressupõe o cuidado integral, contemplado a partir do trabalho grupal. 


\section{O grupo como espaço para o cuidado integral}

A integralidade do cuidado compreende ações voltadas para atender as necessidades do sujeito em sua totalidade, não o dividindo em partes ou sistemas isolados. Os elementos que elegeram essa categoria foram, essencialmente, o "impacto nas condições de saúde" dos sujeitos, tanto "físicas" quanto "psíquicas", o que remeteu à integralidade do cuidado.

Pôde-se observar, pelas narrativas de usuários e profissionais, questões relacionadas às condições físicas que denotaram as implicações do grupo na saúde dos participantes, destacando-se, também, como motivos para a adesão e a permanência às ações grupais.

[...] tinha perda de urina, quando eu tossia. Agora melhorei. [...] o problema do engasgo [...]. (Safira)

[...] exercícios que nós fizemos aqui nos ajudam no equilíbrio [...], e também a parte motora, ativa os músculos [...]. (Quartzo)

[...] além de fazer atividade física, porque eu preciso, porque estou acima do peso [...]. (Mira)

Notou-se a melhora das perdas urinárias, da disfagia, do equilíbrio, da parte muscular e a diminuição do peso. Percebeu-se que houve impacto, não apenas na saúde dos usuários, mas também na dos profissionais.

As narrativas, que seguem, também, denotaram aspectos relacionados às condições álgicas:

Eu passava tomando remédio para a coluna [...]. Nunca mais tomei [...]. Melhorei bastante. [...]. Melhora da qualidade de vida. (Jaspe)

[...] doía tudo aqui, eu não conseguia me abaixar para fazer minhas atividades, agora eu já consigo com mais facilidade [...]. (Diamante)

[...] os dias que eu venho fazer os exercícios, são as noites que eu melhor durmo, porque me dói menos as pernas, me dói menos tudo [...]. (Pérola)

Ah, eu sentia muita dor, e agora não, agora eu estou bem [...]. (Malaquita)

[...] porque eu tenho um problema na coluna, então, é muito bom, ajuda bastante [...] melhora a dor [...]. (Sirius)

Houve melhora em aspectos relacionados à dor e diminuição da medicação para esta, com impacto na qualidade de vida, nas atividades de vida diária (AVDs) e na qualidade do sono. Nesse sentido, as ações de promoção da saúde impactaram sobre as condições clínicas dos usuários, para além das sociais e afetivas demonstradas nas categorias anteriores.

Em um estudo, Santos et al..$^{22}$ discutiram que os grupos de promoção da saúde são capazes de intervir na erradicação ou, ainda, minimizar as doenças e a perda da capacidade funcional dos indivíduos e preservar ou desenvolver a autonomia por parte deles. A AB cumpre com seus princípios na medida em que desenvolve ações em grupos de promoção da saúde dos participantes.

$\mathrm{Na}$ pesquisa realizada por Meurer et al. ${ }^{25} \mathrm{em}$ Centros de Saúde do município de Florianópolis-SC, que teve como objetivo investigar a associação de fatores motivacionais com a autoestima de idosos para a prática de exercícios físicos, os resultados identificaram que os participantes tinham autoestima elevada e sentiam-se motivados pela prática de exercícios físicos, especialmente, nas dimensões da saúde e da sociabilidade ${ }^{25}$. Percebeu-se, tanto no estudo citado ${ }^{25}$ como na presente pesquisa, que as repercussões na saúde se constituíram como importante fator capaz de motivar os sujeitos às práticas grupais. 
Lopes et al. ${ }^{12}$, em uma pesquisa com grupos de convivência em Florianópolis (SC), que se propôs a analisar os motivos de adesão e de permanência de idosos longevos em programas de atividades físicas, obteve como resultados a promoção/manutenção da saúde ${ }^{12}$. Tal resultado ${ }^{12}$ corroborou com o que foi encontrado no estudo aqui apresentado, em que a percepção da saúde é mencionada pelos participantes como importante fator motivacional nas ações grupais.

Nas narrativas que seguem, perceberam-se, também, questões relacionadas à saúde psíquica dos indivíduos:

[...] eu andava meio depressiva, [...] depois que vim minha vida deu uma melhorada cem por cento. (Ágata)

A acolhida no grupo é muito boa [...] é um entrosamento muito bom, levanta o astral da gente [...]. (Cristal)

[...] também na parte de saúde mental, a gente vinha super sufocada com os problemas que a gente não consegue dar muita resolutividade e ali era uma forma de dar uma equilibrada na mente da gente [...]. (Meissa)

[...] você aumenta sua autoestima. [...] vindo aqui você já renova [...]. (Apatita)

É muito bom, porque os exercícios para fazer, minha cabeça precisa [...]. (Citrino)

Cabe destacar, conforme os relatos, que o profissional, por vezes, se coloca como profissional, por vezes, como aprendente, e outras, como usuário, o que, também, foi percebido nas categorias anteriores.

Os discursos dos usuários e profissionais inferiram aspectos relacionados à saúde mental, ou seja, a depressão, o humor, o estresse, a autoestima e a memória, todos beneficiados positivamente pelas atividades desenvolvidas no grupo.-

Em uma pesquisa sobre práticas de grupo desenvolvidas por Equipes de Saúde da Família de Belo Horizonte - MG, envolvendo usuários e profissionais, com a finalidade de conhecer e avaliar as práticas de grupo, demonstrou que as ações contribuíram para a diminuição do uso de medicações indicadas para ansiedade e depressão por parte dos usuários ${ }^{18}$. Nos resultados do presente estudo não foram referidos relatos sobre o uso de medicamentos antidepressivos e ansiolíticos, mas existiu uma melhora nos sintomas depressivos e redução de medicamentos utilizados para a dor.

Nesse contexto, dois estudos semelhantes realizados tiveram como objetivo identificar o que motivavam os idosos a participarem de programas de atividade física e encontraram que um dos principais motivos de adesão e permanência foi a superação do estresse ${ }^{26,27}$. Outra pesquisa realizada com um grupo de convivência de Belo Horizonte - MG, com o intuito de verificar as principais motivações à realização de atividade física em um grupo de mulheres idosas demonstrou que um dos motivos era o aumento da autoestima ${ }^{28}$. A diminuição do estresse e o aumento da autoestima, conforme os relatos dos sujeitos e as pesquisas citadas ${ }^{26-28}$ constituíram importantes fatores de motivação de tais sujeitos no contexto do grupo.

No processo de envelhecimento, o desempenho físico e social de pessoas idosas está intimamente ligado à integridade das funções cognitivas ${ }^{29}$. A partir dos relatos, a memória foi citada como um dos fatores motivacionais, no entanto, não foram encontrados estudos sobre motivos de adesão e permanência que mencionassem esse tema. Entretanto, percebeu-se que em práticas de grupo deve-se ter uma atenção para este aspecto, já que uma alteração na memória pode afetar a funcionalidade dos sujeitos e isto tem sido tema de atenção de profissionais de saúde. 


\section{Conclusão}

Com base nos resultados da pesquisa, percebeu-se que as motivações para o desenvolvimento de ações em grupo na $A B$, na percepção uníssona de usuários e profissionais, compreenderam três categorias, sendo estas: as relações interpessoais, que denotaram as relações de "vínculo" e os "laços afetivos" entre os sujeitos no grupo; a construção de saberes, que representou a "troca de experiências", a "aprendizagem" e o "empoderamento do usuário" quanto ao cuidado com sua saúde e o cuidado integral, que se referiu ao impacto que as ações possuíam nas "condições de saúde" dos sujeitos, tanto "físicas" quanto "psíquicas".

As motivações, aqui encontradas, demonstraram que a atividade grupal compreendeu um importante meio para a promoção da saúde neste nível de atenção, com impacto positivo sobre as condições clínicas, sociais e afetivas dos sujeitos e, consequentemente, na qualidade de vida dessas pessoas.

Assim, pôde-se inferir que os achados do estudo constataram a importância das ações grupais para a promoção da saúde dos usuários, assim como revelaram um dispositivo em potencial para o cuidado integral no nível primário de atenção. Ficou evidente que as ações grupais representaram um espaço para a interlocução do fisioterapeuta em parceria com os profissionais da $A B$.

Diante do exposto, ressalta-se que é importante o desenvolvimento de mais pesquisas sobre o tema, com o propósito de ampliar o diálogo, bem como motivar os profissionais da $A B$ quanto às ações coletivas, preconizadas para esse nível de atenção, em prol de maior abrangência do trabalho interprofissional, assim como da maior resolubilidade das ações realizadas.

\section{Colaboradores}

Thaís Lopes Friedrich, Xavéle Braatz Petermann, Silvana Basso Miolo e Hedioneia Maria Foletto participaram ativamente da discussão dos resultados, da revisão e da aprovação da versão final do trabalho.

\section{Referências}

1. Ministério da Saúde (BR). Portaria n².488, de 21 de outubro de 2011. Aprova a Política Nacional de Atenção Básica estabelecendo a revisão de diretrizes e normas para a organização da Atenção Básica, para a Estratégia Saúde da Família (ESF) e o Programa de Agentes Comunitários de Saúde (PACS). Diário Oficial da União. Brasília, 21 out. 2011. Seção 1, n 204, p. 1-37.

2. Bispo Júnior JP. Fisioterapia e saúde coletiva: desafios e novas responsabilidades profissionais. Cienc Saude Colet. 2010; 15(1):1627-36.

3. Delai KD, Wisniewski MSW. Inserção do fisioterapeuta no programa saúde da família. Cienc Saude Colet. 2011; 16(1):1515-23.

4. Rezende M, Moreira MR, Amancio Filho A, Tavares MFL. A equipe multiprofissional da "Saúde da Família": uma reflexão sobre o papel do fisioterapeuta. Cienc Saude Colet. 2009; 14(1):1403-10. 
5. Formiga NFB, Ribeiro KSQS. Inserção do fisioterapeuta na atenção básica: uma analogia entre experiências acadêmicas e a proposta dos núcleos de apoio à saúde da família (NASF). Rev Bras Cienc Saude. 2012; 16(2):113-22.

6. Carvalho STRF, Caccia-Bava MCGG. Conhecimentos dos usuários da estratégia saúde da família sobre a fisioterapia. Fisioter Mov. 2011; 24(4):655-64.

7. Baena CP, Soares MCF. Subsídios reunidos junto à equipe de saúde - a inserção da fisioterapia na estratégia saúde da família. Fisioter Mov. 2012; 25(2):419-31.

8. Aquino CF, Augusto VG, Moreira DS, Ribeiro S. Avaliação da qualidade de vida de indivíduos que utilizam o serviço de fisioterapia em unidades básicas de saúde. Fisioter Mov. 2009; 22(2):271-9.

9. Santos LM, Oliveira EM, Crepaldi MA, Ros MA. Atuação dos coordenadores de grupos de saúde na rede docente assistencial. Rev Saude Publica. 2010; 44(1):177-84.

10. Santos LM, Ros MA, Crepaldil MA, Ramos LR. Grupos de promoção à saúde no desenvolvimento da autonomia, condições de vida e saúde. Rev Saude Publica. 2006; 40(2):346-52.

11. Combinato DS, Vecchia MD, Lopes EG, Manoel RA, Marino HD, Oliveira ACS, et al. "Grupos de Conversa": saúde da pessoa idosa na estratégia da saúde da família. Psicol Soc. 2010; 22(3):558-68.

12. Lopes MA, Krug RR, Marches M, Mazo GZ. Análise da aderência e da permanência de longevos em programas de atividade física. ConScientiae Saude. 2012; 11(3):429-37.

13. Minayo MCS. O desafio do conhecimento: pesquisa qualitativa em saúde. 12a ed. São Paulo: Hucitec; 2010.

14. Fontanella BJB, Ricas J, Turato ER. Amostragem por saturação em pesquisas qualitativas em saúde: contribuições teóricas. Cad Saude Publica. 2008; 24(1):17-27.

15. Augusto VG, Aquino CF, Machado NC, Cardoso VA, Ribeiro S. Promoção de saúde em unidades básicas: análise das representações sociais dos usuários sobre a atuação da fisioterapia. Cienc Saude Colet. 2011; 16(1):957-63.

16. Mattos $S M N$. Avaliar: um diálogo da afetividade com a rede de relações desenvolvidas no cotidiano escolar. Rev Educ PUC-Camp. 2008; 25:173-81.

17. Bittar C, Lima LCV. O impacto das atividades em grupo como estratégia de promoção da saúde na senescência. Rev Kairos. 2011; 14(3):101-18.

18. Ferreira Neto JL, Kind L. Práticas grupais como dispositivo na promoção da saúde. Physis. 2010; 20(4):1119-42.

19. Maffacciolli R, Lopes MJM. Os grupos na atenção básica de saúde de Porto Alegre: usos e modos de intervenção terapêutica. Cienc Saude Colet. 2011; 16(1):973-82.

20. Coelho MO, Jorge MSB. Tecnologia das relações como dispositivo do atendimento humanizado na atenção básica à saúde na perspectiva do acesso, do acolhimento e do vínculo. Cienc Saude Colet. 2009; 14 Supl 1:1523-31.

21. Krug RR, Lopes MA, Mazo GZ. Barreiras e facilitadores para a prática da atividade física de longevas inativas fisicamente. Rev Bras Med Esporte. 2015; 21(1):57-64.

22. Viegas $S M F$, Penna $C M M$. A construção da integralidade no trabalho cotidiano da equipe de saúde da família. Esc Anna Nery. 2013; 17(1):133-41.23. Almeida SP, Soares SM. Aprendizagem em grupo operativo de diabetes: uma abordagem etnográfica. Cienc Saude Colet. 2010; 15 (1):1123-32.

24. Taddeo PS, Gomes KWL, Caprara A, Gomes AMA, Oliveira GC, Moreira TMM. Acesso, prática educativa e empoderamento de pacientes com doenças crônicas. Cienc Saude Colet. 2012; 17(11):2923-30. 
25. Meurer ST, Benedetti TRB, Mazo GZ. Teoria da autodeterminação: compreensão dos fatores motivacionais e autoestima de idosos praticantes de exercícios físicos. Rev Bras Ativ Fis Saude. 2011; 16(1):18-24.

26. Cavalli AS, Pogorzelski LV, Domingues MR, Afonso MR, Ribeiro JAB, Cavalli MO. Motivação de pessoas idosas para a prática de atividade física: estudo comparativo entre dois programas universitários - Brasil e Portugal. Rev Bras Geriatr Gerontol. 2014; 17(2):255-64

27. Ribeiro JAB, Cavalli AS, Cavalli MO, Pogorzelski LV, Prestes MR, Ricardo LIC. Adesão de idosos a programas de atividade física: motivação e significância. Rev Bras Med Esporte. 2012; 34(4):969-84.

28. Gomes KV, Zazá DC. Motivos de adesão à prática de atividade física em idosas. Rev Bras Ativ Fis Saúde. 2009; 11(2):132-8.

29. Ministério da Saúde (BR). Secretaria de Atenção à Saúde. Departamento de Atenção Básica. Envelhecimento e saúde da pessoa idosa. Brasília: Ministério da Saúde; 2006.

Friedrich TL, Petermann XB, Miolo SB, Pivetta JMF. Motivaciones para prácticas colectivas en la Atención Básica: percepción de usuarios y profesionales. Interface (Botucatu). 2018; 22(65):373-85.

El objetivo de este estudio fue comprender las motivaciones para el desarrollo y el mantenimiento de acciones grupales en la Atención Básica en la percepción de usuarios y profesionales. Se trató de una encuesta descriptiva con metodología cualitativa desarrollada en una Unidad de Salud con Estrategia de Agentes Comunitarios de Salud de un municipio del Sur de Brasil. Participaron 15 usuarios y cinco profesionales, siendo la muestra intencional por conveniencia y saturación. Los datos se colectaron por medio de entrevista narrativa, semi-estructurada individual y se analizaron por medio del análisis de contenido. Las categorías que denotaron las motivaciones de los sujetos incluyeron las relaciones interpersonales, la construcción de saberes y el cuidado integral. Los resultados encontrados retrataron la importancia del trabajo grupal en la Atención Básica para la promoción de la salud, una vez que presentaron impacto positivo sobre las condiciones clínicas, sociales y afectivas de los sujetos.

Palabras clave: Atención primaria de la salud. Procesos grupales. Motivación. 
